\title{
Shining a light on photochemical dynamics
}

When a molecule absorbs light, this can trigger a whole host of reactions. This includes the formation of new bonds or even rearranging the order of the atoms within the same molecule to create light-induced changes, know as photochemical dynamics, can be challenging to study as they can occur on timescales of femtoseconds, so fast that even the quickest camera would struggle to keep up. Dr Art Bragg and his research group at John Hopkins have been addressing this by using ultrafast laser spectroscopy to gain now reactions occur. ow molecules interact with light employed by synthetic chemists to make plays an important part in our daily
lives. One of the ways we perceive the colour of an object is because it absorbs all other wavelengths of light, reflecting only the colour we consider it to be. However, sometimes the packets of light energy we call photons - that not so to see colours and objects - are may actually trigger reactions that alter the chemical structure of a species.

One common example of this is how paints and fabrics lose their colour and degrade when exposed to light. As the molecules absorb photons and their energy over time, they start to break down and form new chemical species with different optical properties than the original molecule.

However, interacting with light doesn't have to be destructive for molecules. Some molecules use light energy as the kick photochemistry is a common trick. formed by conventional methods, like heating, alone.

Dr Art Bragg and his team at John Hopkins are using state-of-the-art laser processes to understand exactly what photons. By using pulsed femtosecond lasers, capable of producing bursts of photons that last for just quadrilionths of a second he has pieced together detailed mechanisms of how molecula species are changed by light.

\section{PHOTOCHEMISTRY}

Dr Bragg of research projects emtosecond spectroscopic techniqus to understand photochemical bond formation reactions in exquisite levels of detail. In particular, he has explored a class of chemical reactions known as photoinduced electrocyclisation reactions. These are a broad family

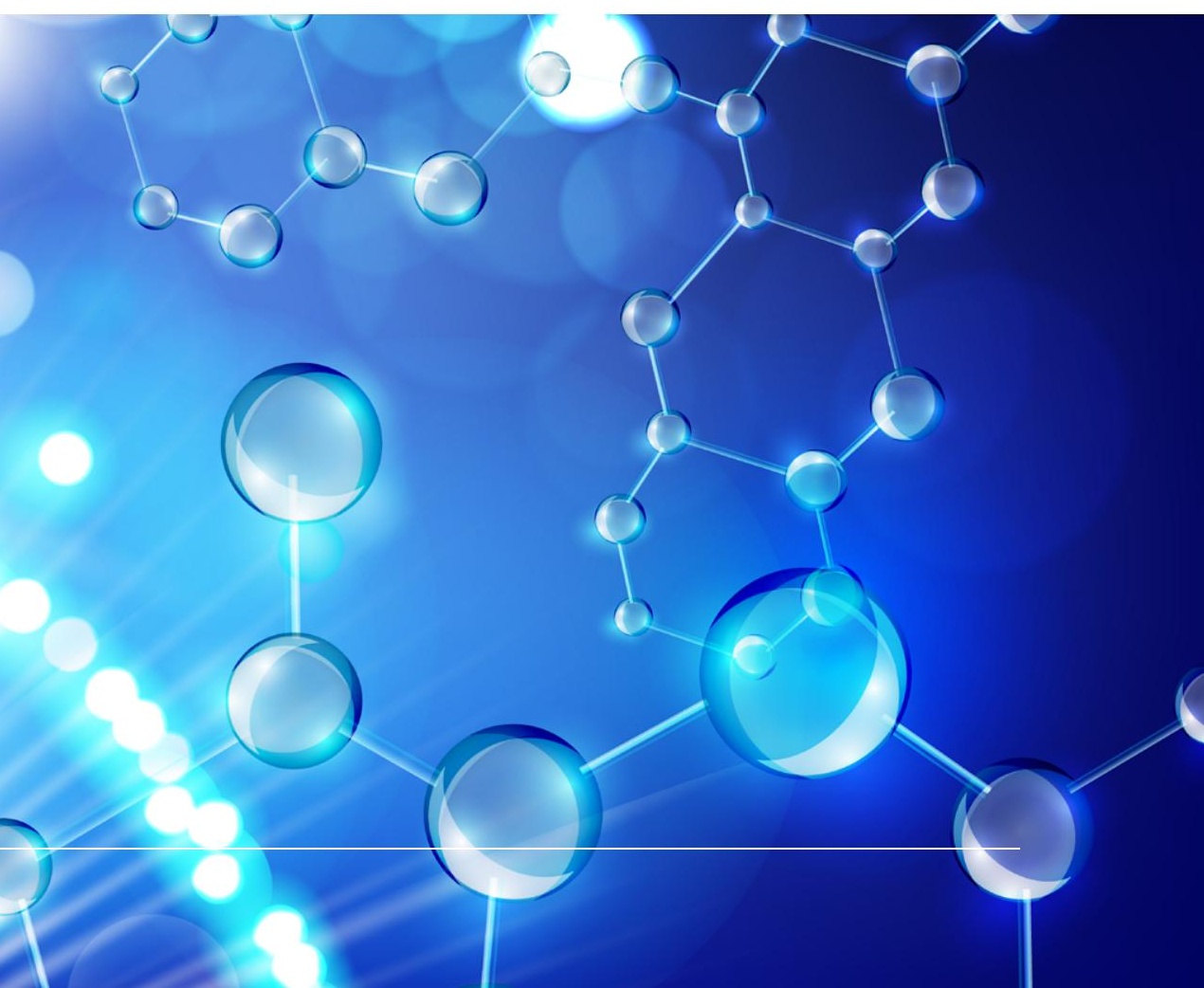

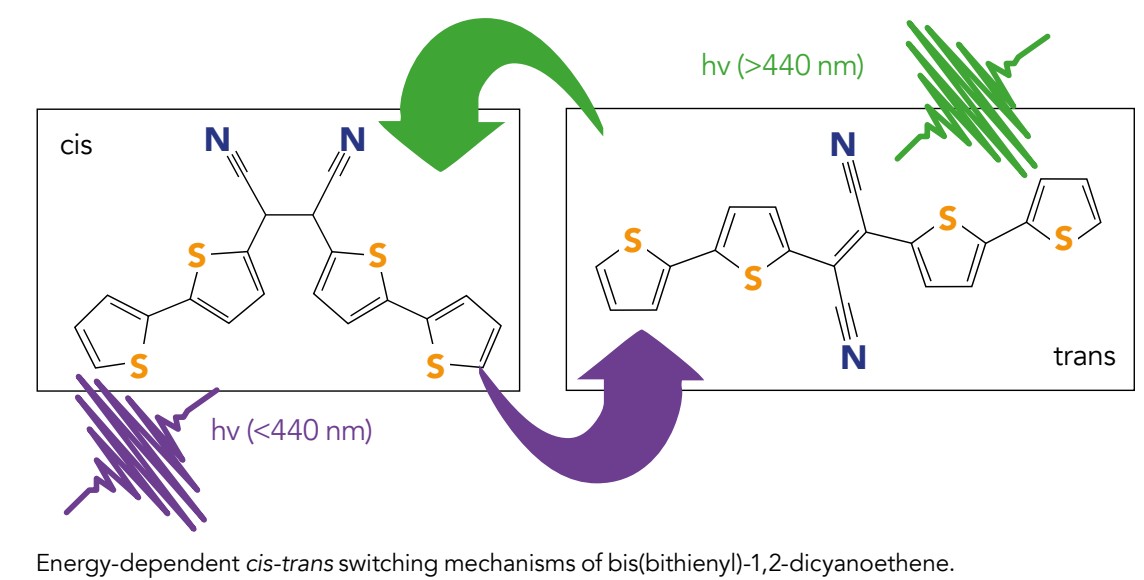

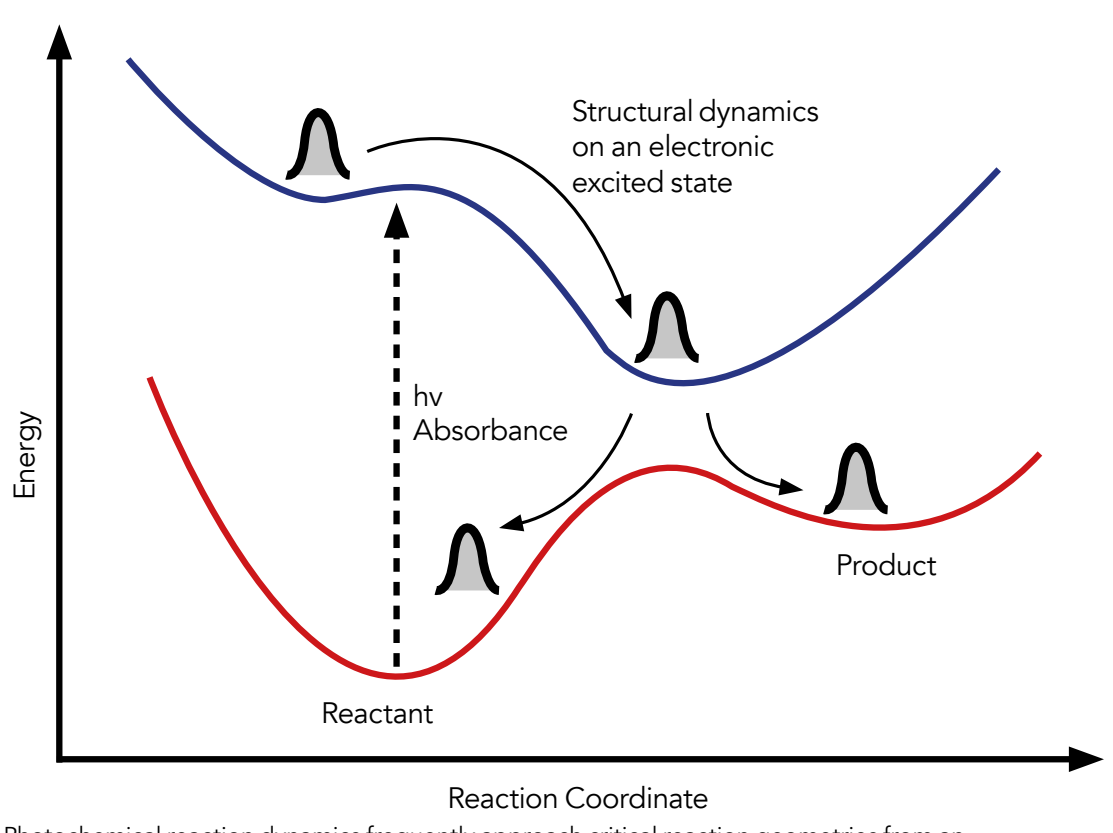

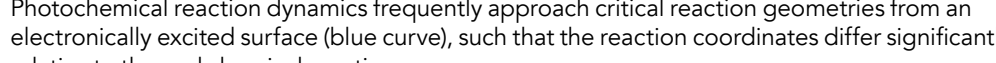

either within a molecule or between

two or more different orolecteen two or more different molecules, key intermediate structures in a photocyclisation reaction. Through bonds in a joined ring. This chemical structure is at the heart of many useful compounds, including polyaromatic dectures, Dr Bragg has the starting molocute to influence the final products of the photoinduced electrocyclisation reaction. Ultimately,

hydrocarbons, valuable intermediates

(1)

Dr Art Bragg and his team are using state-of-the-art laser technology to understand exactly what processes occur after molecules absorb photons.

in chemical synthesis of many compounds, including pharmaceuticals. this involves changing how the molecules Using a combination of ultrafast spectroscopy and quantum chemical
calculations, Dr Bragg has identified

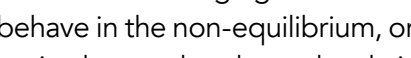
excited, state that the molecule is in Finter the photon absorption process. an important step in being able to boost product vields for light-induced synthesis of important compounds.

\section{PHOTOSWITCHING}

In addition to driving formation of new bonds, light absorption can also drive a change in the overall shape of a molecule a process known as photoisomerisation. The structural rearrangement of the
positions of the atoms in a molecule happens because certain configurations are stabilised in the excited state which may be energetically inaccessible in the unperturbed ground state of the molecule. This enables molecules to reach new structures following relaxation from these higher-energy states. Dr Bragg has been focusing on the photoisom risation processes of hecur different stable ger that can be interconverted when they absorb light While the two forms of the molecule have all the same atoms and are structures that normally differ by a twist around one bond, they can have very different properties, including absorbing light at such different wavelengths that the two forms of the molecule are different colours.

One photoswitch that exhibits poor switching efficiency has been particularly interesting to Dr Bragg's group. The team have found that this photoswitch has different conformers (which are slight structura variations of the same jomel. In tum, hection very dfferent relaxation dynamics, only Furthermore isomeristion efficiency of this switch is impacted by which specific excited state of the molecule is reached by light absorption and the details of the cascade of processes, resulting in its deactivation. When a molecule absorbs excess energy from a photon, this new non-equilibrium state is typically unstable and so the molecule will try to find ways to dispose of this excess energy. To do this might involve conversion between different structures, transfer of energy to vibrations of bonds or in the case of molecules in solution, by losing energy to the surrounding Hown molecules through collisions. How energetic saxation occurs and some mocus ilie those inendent; 
